\title{
A Computer-Aided Detection of EEG Seizures in Infants: A Singular-Spectrum Approach and Performance Comparison
}

\author{
Patrick Celka*, Member, IEEE, and Paul Colditz
}

\begin{abstract}
This paper presents a scalp electroencephalogram (EEG) seizure detection scheme based on singular spectrum analysis (SSA) and Rissanen minimum description length (MDL) model-order selection (SSA-MDL). Preprocessing of the signals allows for the drastic reduction of the number of false alarms. Statistical performance comparison with seizure detection schemes of Gotman et al. and Liu et al. is performed on both synthetic data and real EEG seizures. Monte Carlo simulations based on synthetic infant EEG seizure data reveals some detection drawbacks on a large variety of seizure waveforms. Detection using both Monte Carlo and four real infant scalp EEG signals shows the superiority of the SSA-MDL method with an average good detection rate of $>93 \%$ and false detection rate $<4 \%$.
\end{abstract}

Index Terms-Detection, EEG seizure, infant, MDL, newborn, singular spectrum analysis.

\section{INTRODUCTION}

A BNORMALITIES in the electroencephalogram (EEG) have a good predictive value for a poor neurodevelopmental outcome in the newborn and infant [1]. Because the duration of the potential therapeutic window, for the use of neural rescue agents, is about 2-6 hours [2], automatic detection of predefined patterns have started to be investigated. Seizure EEG patterns have been studied using computerized methods. This subclass of the so-called paroxysmal-type EEG patterns has been shown to provide reliable predictive indicators for encephalopathy. In most cases, infants showing seizure have poor health outcomes and a great probability of death [3].

To the best of our knowledge, two efficient methods have been developed and thoroughly assessed for computer-aided detection of seizures in newborn and infant scalp EEG signals. The first method is based on the computation of a running autocorrelation function and was proposed by Liu et al. [4] (LIU). The second method, proposed by Gotman et al. [5] (GOTMAN), is based on the analysis of running periodograms. We would like to point out that detection of EEG events in newborn and infants cannot be performed without a close inspection of many

Manuscript received July 9, 2001; revised December 12, 2001. This work was supported by grants from the Australian Research Council, SPIRT, and ARC grants. Asterisk indicates corresponding author.

*P. Celka was with the Signal Processing Research Centre, Queensland University of Technology, Brisbane Qld 4001, Australia. He is now with the Swiss Center for Electronics and Microtechnology, Systems Engineering Division, Rue Jaquet-Droz 1, CH-2007 Neuchâtel, Switzerland (e-mail: patrick.celka@csem.ch).

P. Colditz is with the Perinatal Research Centre, The University of Queensland, Royal Women's Hospital, Brisbane Qld 4029, Australia.

Publisher Item Identifier S 0018-9294(02)03998-8. signals such as EEG electrocardiogram, respiratory excursions, electro-occulogram, and video. This is because daily care of babies can produce EEG waveforms that mimics typical EEG patterns, and artifacts/interferences can mask the relevent information. For these reasons, we believe that detection of EEG patterns in infants cannot be fully automated and we prefer using the terminology computer-aided detection.

A new seizure detection method based on singular spectrum analysis (SSA) and information theoretic-based selection of the signal subspace is designed in this paper [SSA-Rissanen minimum description length (MDL) model-order selection (SSA-MDL)]. This approach is shown to outperform the above mentioned detection schemes (LIU and GOTMAN). The motivations for using the SSA are: 1) SSA performs very well on quasi-periodic signals, which is the case for EEG seizures and 2) the use of singular-value decomposition (SVD) of the so-called trajectory matrix is highly robust to noise. The detection scheme proceeds with a preprocessing of the data, SSA and the use of Rissanen's MDL criterion [6], [7]. The preprocessing is based on a nonlinear whitening filter that spreads the spectrum of the background while keeping rhytmical features of the seizure events. The nonlinear function transform the non-Gaussian shape of the probability density function (pdf) of the EEG into a Gaussian one. This allows for the optimal use of MDL and reduces the effects of the artifacts. Using such a criterion also reduces the drawback of using subjective and data-dependent predefined threshold, typical of classical test-statistic detectors.

EEGs from newborns and infants varie from day to day and displays: 1) nonstationarity during a single recording [8]-[11]; 2) a non-Gaussian pdf [12]; 3) various artifacts; and 4) a rhythmical background EEG for which the frequency spectrum largely overlap with the seizure one. These signal characteristics may impinge on the performances of computer-based detection, and motivates the assessment of published methods. The easiest and most reliable way to do this assessment is to generate synthetic EEG signals with prescribed background and seizure. We used synthetic data of EEG seizures presented in [13].

This paper is organized as follows. Section II describes the data acquisition method. Section III presents the nonlinear nonstationary model-based EEG seizure scheme. Section IV introduces the SSA-MDL detection method, along with LIU and GOTMAN. Section V presents the results of the statistical performance analysis of LIU, GOTMAN, and SSA-MDL on synthetic data. Section VI compare the performances of the three 


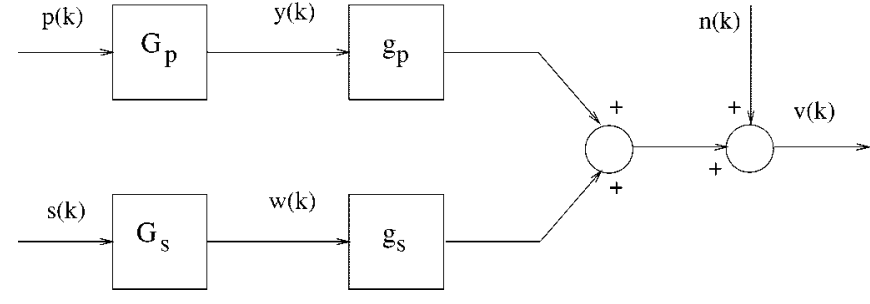

Fig. 1. Nonlinear nonstationary model of EEG seizure.

detection schemes on ten babies suffering from EEG seizures. Section VII discusses the results. Conclusions are presented in Section VIII

\section{EXPERIMENTAL SETUP}

The data acquisition was performed at the Royal Women's Hospital and Royal Children's Hospital, Brisbane, Australia. Between five and 20 EEG channels were recorded depending on the available recording system and the head size. Four babies from conceptual age (between five and seven weeks) to maximum six months after birth were used.

$\mathrm{Ag}-\mathrm{AgCl}$ electrodes flushed with conductive gel and adhered by tape attached to the skin of the infant were used [14]. The four babies were showing signs of clinical and electrical seizures. These four recordings were visually segmented (extraction of the seizure epochs) by a neurologist from the Neurosciences Department at the Royal Children's Hospital.

The electrode placement agrees with the American EEG Society standards, while the electrode positions F1 and F2 are not true 10-20 positions, but are commonly used for babies [14]. Slow baseline fluctuations due to baby movement have been removed by using a second-order high-pass Butterworth filter with a cutoff frequency of $0.1 \mathrm{~Hz}$. These signals were amplified and digitized using either the Amlab (AmLab Technologies, Lewisham, NSW, Australia) or Medelec (Oxford Instruments, U.K.) software/hardware environment. The sampling frequency was set to $F_{s}=256 \mathrm{~Hz}$. EEG signals were then subsampled at $F_{r}=40 \mathrm{~Hz}$ to agree with GOTMAN [5] and LIU [4] detection standard.

Surface electrocardiogram (three leads), a symmetric electro-occulogram, and respiratory excursions signals were also recorded for control purposes.

\section{Synthetic EEG SEIZURE}

In order to compare the performance of the SSA-MDL algorithm with LIU and GOTMAN, we used the EEG model proposed in [13] to generate synthetic background and seizure EEG activities. The model structure, shown in Fig. 1 and detailed in [13], is derived from a previously proposed seizure model by Roessgen et al. [15] who extended the model initiated by Lopes da Silva et al. [16] in introducing a seizure input sawtooth signal $z(t)$. An identification procedure has been examined in [13]. It is assumed throughout the text that the signals are sampled such that the continuous-time variable $t$ is discretized as $t=k / F_{r}$, and we use $t$ or $k$ depending on the context. The pure background activity is modeled by an autoregressive moving average
(ARMA) filter $G_{p}(z)$ excited by a zero mean GWN $p(k)$, followed by a nonlinear function $g_{p} . p(k)$ is assumed to model deep brain activities in structures such as thalamus and brain stem. In parallel to this branch, an other ARMA filter $G_{s}(z)$ is excited by a deterministic signal $s(k)$ followed by a nonlinear function $g_{s}$. The signal $s(k)$ is a piecewise linear frequency modulated sawtooth signal [13]. The later branch is expected to represents the pure seizure activity. The sum of these two branches gives the output signal $v(k)$, which is also the measured EEG signal, and expressed by

$$
v(k)=g_{s}[w(k)]+g_{p}[y(k)]+n(k)
$$

where $y(k)=G_{p}(z) p(k)$ and $w(k)=G_{s}(z) s(k)$. A measurement noise $n(k)$, assumed to be Gaussian and white (GWN) of variance $\sigma_{n}^{2}$ and zero mean, is added. The input signals are the GWN $p(k)$ and the deterministic signal $s(t)$ expressed as

$$
s(t)=z(t) e^{j 2 \pi f_{i}(t) t}, \text { where } f_{i}(t)=\frac{\alpha}{2} t
$$

$f_{i}(t)+f_{o}$ is the instantaneous frequency of $s(t)$ with $z(t)$ a sawtooth signal of period $1 / f_{o}$. In the full model proposed in [13], $f_{i}(t)$ is a three-element piecewise linear function. But, for our performance comparison, a simple linear frequency modulated law is sufficient. The $\alpha$ parameter represents the slope of linear frequency modulation. The output signal $v(k)$ mean is set to zero and its variance normalized to unity. The two last terms on the right-hand side of (1) can be interpreted as the stochastic parts of the model and grouped as $\eta(k)=g_{p}[y(k)]+n(k)$ such that $v(k)=g_{s}[w(k)]+\eta(k)$. In most of the situations, the contribution $\eta(k)$ to the total EEG activity is less important than the pure seizure activity $g_{s}[w(k)]$ resulting in a relatively high seizure-to-background ratio (SBR)

$$
\mathrm{SBR}=10 \log _{10} \frac{E\left[g_{s}^{2}[w(k)]\right]}{E\left[\eta^{2}(k)\right]+\sigma_{n}^{2}} .
$$

Using SSA and model selection [17], we have estimated that $10 \mathrm{~dB} \leq \mathrm{SBR} \leq 30 \mathrm{~dB}$ on $56 \mathrm{EEG}$ seizure segments. Assuming the independence of $s(k)$ and $p(k)$, the signal-to-noise ratio (SNR) is given by

$$
\mathrm{SNR}=10 \log _{10} \frac{E\left[g_{s}^{2}[w(k)]\right]+E\left[g_{p}^{2}[y(k)]\right]}{\sigma_{n}^{2}}
$$

Fig. 2 shows recorded and synthetic background activities, and Fig. 3 shows recorded and synthetic seizure activities. We have used SNR $=20 \mathrm{~dB}, \mathrm{SBR}=20 \mathrm{~dB}$. The model was identified from a baby displaying EEG seizures as in [13]. Note that the seizure signal in Fig. 3 is nonstationary and nonsymmetrical in amplitude, and the synthetic data do reproduce those behaviors.

We have selected four parameters that are mostly susceptible to influence the detection performances of LIU, GOTMAN, and SSA-MDL: the frequency $f_{o}$ (especially for GOTMAN), the slope $\alpha$ which specifies the degree of nonstationarity, the SNR, and the SBR. 

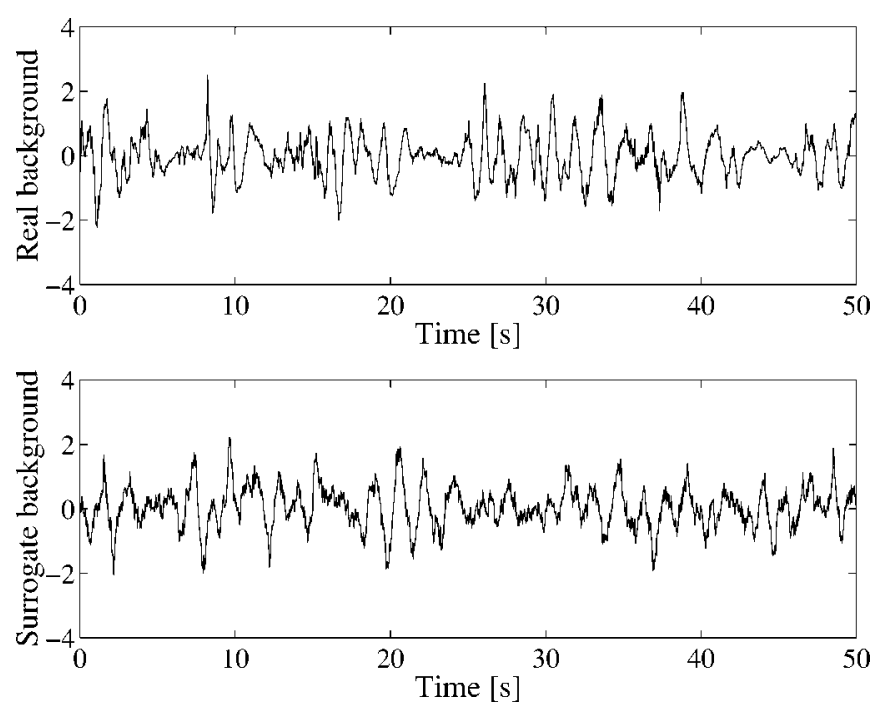

Fig. 2. Examples of real and synthetic background EEG activities.

\section{Detection Techniques}

\section{A. SSA-MDL Method}

1) Singular Spectrum Analysis: Neurons and neuronal networks composing the central nervous system can discharge in both asynchronous or synchronous manners. Asynchronous discharges lead to a continuous background activity while synchronous activity leads to rhythmical patterns such as seizures [8], [18] (peaked power spectral density). It is well known that SSA is particularly suited for extracting information from quasi-periodic signals embedded in noise [19]. SSA has been used in nonlinear time series analysis with more or less success [20]-[24], but has also been shown to provide interesting results in biomedical applications [25]-[28].

The measured EEG signal $\mathbf{x}=\{x(k)\}_{k=1}^{N}$ is zero-meaned and normalized to have unit variance. Let $\mathrm{x}_{k}=[x(k), x(k+$ $\left.J * 1), \ldots, x\left(k+J *\left(n_{s}-1\right)\right)\right]^{T}$ be a state vector in $\mathbb{R}^{n_{s}}$. The trajectory matrix is defined as ${ }^{1}$

$$
Z^{T}=\left[\mathrm{x}_{1} \mathrm{x}_{2} \cdots \mathrm{x}_{N_{T}}\right]
$$

where $N_{T}=N-\left(n_{s}-1\right)$. The size of the trajectory matrix $Z$ is $N_{T} \times n_{s}$. The trajectory matrix may be viewed as a cloud of points in $\mathbb{R}^{n_{s}}$ to which an $n_{o}$-dimensional ellipsoid can be fitted. The $n_{O}$ principal axes of this ellipsoid are given by the eigenvectors $\mathbf{e}_{i}$ of the covariance matrix $A_{z z}=\left(Z^{T} Z\right) / N_{T}$ corresponding to the $n_{0}$ largest eigenvalues from $\lambda_{1} \geq \lambda_{2} \geq$ $\cdots \lambda_{n_{o}} \geq \cdots \lambda_{n_{s}}$. The maximum number of eigenvalues is $a$ priori given by $n_{s}=n_{o}+\left(n_{s}-n_{o}\right)$. The signal $\mathrm{x}$ can eventually be separated in two parts: signal (the deterministic part) and noise (the stochastic part) which are related to the $n_{O}$ first and $n_{s}-n_{O}$ last eigenvalues. The choice of $n_{s}$ is crutial and it has been shown in [19] that, for quasi-periodic signals, an upper bound is given by $n_{s}<\min \left\{F_{r} / \Delta B,(N / 3+1)\right\}$ where $\Delta B$ is the bandwidth of the information bearing signal, for instance the seizure.

Instead of computing the sample covariance matrix $A_{z z}$, we performed the SVD on $Z$. The reason for that choice is the

${ }^{1}$ We used a unit delay $J=1$ while other delays may also be used.
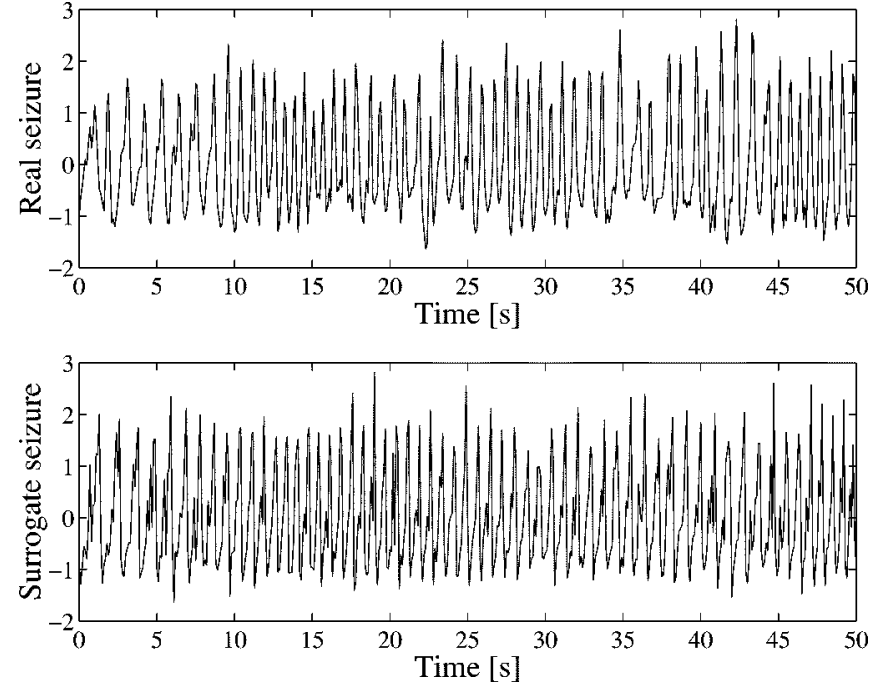

Fig. 3. Examples of real and synthetic seizure EEG activities.

robustness of the SVD against noise and its efficiency in estimating the eigenvalues of $A_{z z}$ for short time series. The singular values $\sigma_{i}$ of $Z$ satisfies $\sigma_{i}^{2}=\lambda_{i}$ for $i=1, \ldots, n_{s}$.

2) Minimum Description Length: The crucial question which now arises is how to determine $n_{o}$. The state space is of dimension $n_{s}$ and is supposed to contain the minimal size embedding space of dimension $n_{0} \leq n_{s}$. The goal now is to perform a dynamical information bearing subspace extraction; or, in other words, an optimal dimension estimation. From previous works [26]-[28], it apppears that the Rissanen's MDL criterion is well adapted to the case of subspace selection in noisy environments. The formula of the MDL criterion is given by [25]

$$
\begin{aligned}
\operatorname{MDL}\left(l, \alpha_{i}\right)= & -\ln \left[\frac{\prod_{i=l+1}^{n_{s}} \alpha_{i}^{1 /\left(n_{s}-l\right)}}{\frac{1}{n_{s}-l} \sum_{i=l+1}^{n_{s}} \alpha_{i}}\right]^{N_{T}\left(n_{s}-l\right)} \\
& +n_{f}(l)\left(\frac{1}{2}+\ln [\gamma]\right) \\
& -\frac{n_{f}(l)}{l} \sum_{i=1}^{l} \ln \left[\alpha_{i} \sqrt{\frac{2}{N_{T}}}\right]
\end{aligned}
$$

where $\gamma=32$ corresponds to a floating point representation, and the number of freely adjustable parameters $n_{f}(l)$ is given by $n_{f}(l)=n_{s} l-\left(l^{2} / 2\right)+l / 2+1$. In the case of the $L_{2}$ norm, $\alpha_{i}=\lambda_{i}$, while if we use the $L_{1}$ norm we should use $\alpha_{i}=\sigma_{i}$. The optimal model order $n_{\odot}$ minimizes MDL and is given by

$$
n_{o}=\arg \min _{l \in\left\{1, \ldots, n_{s}\right\}} \operatorname{MDL}\left(l, \alpha_{i}\right) .
$$

The following two situations can be encountered depending on the value of $n_{o}$ :

1) if $n_{o}=1$, then the signal $x$ can be considered as a pure white noise;

2) if $n_{O}>1$, the signal contains a nonstochastic component.

The meaning of $n_{\odot}$ is very important in order to understand the principle of our detection technique. First, note that the min- 
imum number of eigenvectors needed for representing a pure sine wave, which is in some sense the minimal rhythm, is two. Thus, $\left\lfloor n_{0} / 2\right\rfloor(\mid x\rfloor$ is the smallest integer below $\left.x\right)$ is the number of components (or harmonics) in the signal. This component counting property of $n_{O}$ has been explained in [19], [27], and [28]. Second, the usefulness of using SSA is to separate the noise part of the signal from the more deterministic part which is supposed to contain most of the information. $n_{o}$ measure the complexity of the deterministic part of the signal. If the signal is composed of a pure white noise, there is no deterministic part and $n_{o}=1$, otherwise $n_{O}>1$.

We expect that signals for which $n_{0} \gg 3$ are rather complex and most probably originates from a high-dimensional system, which seems unlikely for seizure activity [27], [29]-[32].

The situation where $n_{0} \approx 3$ signifies that the deterministic part of the signal is quasi-periodic, or originates from a lowdimension system and may be used for detection of rhythmic activity.

3) Preprocessing: We want to separate the background from seizure activities as much as possible. We thus preprocess the data in order to meet the condition $n_{O}=1$ in background EEG. The preprocessing makes use of the model presented in Section III. The function $g_{p}$ and the filter $G_{p}$ are estimated (see [13] for details) on some background EEG of the signal to be processed. The estimated inverse nonlinear function $\hat{g}_{p}^{-1}$ is first applied to the measured EEG $x(k)$ in order to Gaussianize the data, then the estimated inverse filter $\hat{G}_{p}^{-1}$ is applied to $x(k)$ and used for whitening the background EEG. The resulting signal $x_{n}(k)$ is

$$
\begin{aligned}
x_{n}(k) & =\hat{G}_{p}^{-1}(z) \hat{g}_{p}^{-1}[x(k)] \\
& =\hat{G}_{p}^{-1}(z)\left(\hat{g}_{p}^{-1} \circ g_{s}\right)\left[G_{s}(z) s(k)\right]+\tilde{n}(k)
\end{aligned}
$$

where $s(k)=0$ for background EEG and $\tilde{n}(k)$ is a Gaussian noise with a broadband continuous spectrum. ${ }^{2}$ The preprocessed signal $x_{n}(k)$ thus contains a deterministic part $\left(\hat{G}_{p}^{-1}(z)\left(\hat{g}_{p}^{-1} \circ g_{s}\right)\left[G_{s}(z) s(k)\right]\right)$, and a stochastic part $(\tilde{n}(k))$. Eventually, the MDL criterion will discriminates between a whitened background activity for which we expect to have $n_{o}=1$ and a seizure activity with $n_{\circ}>1$.

The SSA-MDL detection scheme proceeds in four steps which are summarized in Fig. 4 and described hereafter. First, the signal is preprocessed, then segmented using a sliding window of $10 \mathrm{~s}$ from which $Z$ is constructed. The window proceed by a 1.25 -s step, The SVD of $Z$ is performed, and $n_{0}$ is computed using (6) and (7) with the $L_{1}$ norm. We set a flag $F=1$ if $n_{o}>1$, and $F=0$, otherwise. We finally stack the flags into a vector and apply a median filter of order three in order to remove isolated flags $F=1$.

\section{B. Gotman's Method}

Gotman et al. [33]-[35] presented three separate methods that are intended to be used simultaneously to detect seizure. This allows each method to be developed for specific waveforms with a lower degree of variability. However, since using the three methods together causes an increase in the false detec-

\footnotetext{
${ }^{2}$ It is not perfectly white due to $\hat{G}_{p}^{-1}$.
}

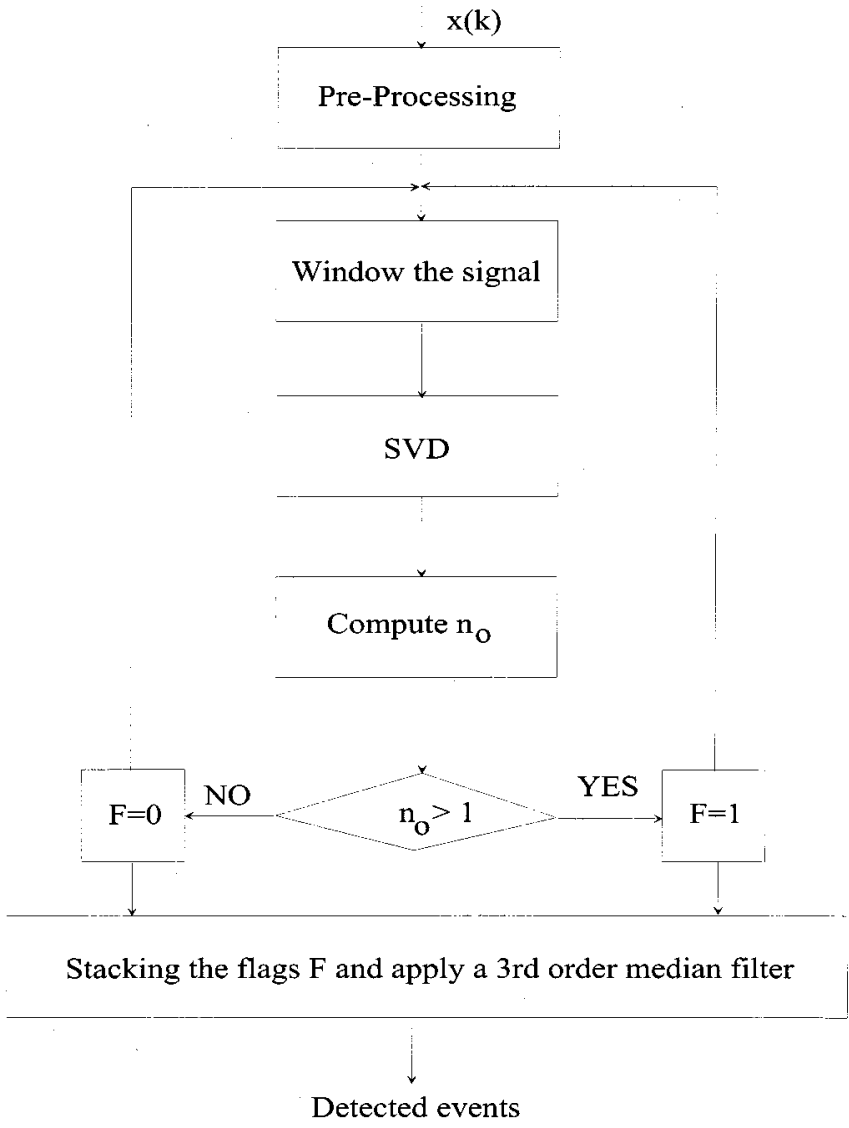

Fig. 4. Structure of the SSA-MDL detection algorithm.

tion rate (FDR), only the first method developed by Gotman will be discussed here. This method was developed specifically for seizure detection in neonates. The other two methods are modified versions of previously developed algorithms for automatic seizure detection in adults [34], [35]. The method described by Gotman [33] is based on spectral analysis and is used to detect periodic discharges. A background epoch is defined as a $20-\mathrm{s}$ segment of EEG finishing $60 \mathrm{~s}$ before the start of the current 10 -s epoch being investigated. The main advantage of a moving background epoch is that results are not dependent on the specific features of a fixed epoch. The frequency spectrum of each 10 -s epoch is calculated and the following features are extracted:

- the frequency of the dominant spectral peak;

- the width of the dominant spectral peak;

- the ratio of the power in the dominant spectral peak to that of the background spectrum in the same frequency band.

The three features are used for seizure detection in each epoch. If an epoch is classified as containing seizure, a further three criteria are used to limit the number of false detections (FDs). Seizure detection is discounted if the epoch is largely nonstationary, if there is a large amount of noise power present or if it appears that an EEG lead has been disconnected.

The aim of this method is to determine if a dominant peak exists in the power spectal density estimate. This is equivalent to detecting if an EEG waveform has a dominant periodic shape in the time domain. The feature space used to classify an epoch as seizure ensures that the dominant peak of the spectrum is significant compared with the background spectrum. 


\section{Liu's Method}

As with the above approaches, the technique of Liu et al. [4] assumes that the essential characteristic in newborn seizure EEG is periodicity. The amount of periodicity in the autocorrelation of short epochs of EEG data is scored and used in a rule-based algorithm to perform classification [4]. In this technique, an epoch consisting of $30 \mathrm{~s}$ of data is divided into five windows. Depending on the autocorrelation function of a window, up to four primary periods $\left(T_{1}, \ldots, T_{4}\right)$ are calculated for each window in an epoch. These times correspond to the times of the moment centers of the first, second, third, and fourth peaks in the autocorrelation function. The windows are then scored, whereby more evenly spaced primary periods are allocated larger scores. After each window in an epoch is scored, a rule-based detection scheme is applied to classify each epoch as positive or negative . If two or more channels of EEG data in the same epoch are positive, the epoch is then classified as containing seizure. For the sake of comparision with the other two techniques, we implemented Liu's method using only one channel.

\section{Performance Comparison on Synthetic Data}

We generated $T=8 \mathrm{~min}$ of background and seizure patterns randomly placed within $T$. The duration $T_{\mathrm{SZ}}$ of the seizure was also set randomly between 5 and $30 \mathrm{~s}$. Only the number $S Z_{>10}$ of seizure patterns for which $T_{\mathrm{SZ}} \geq 10 \mathrm{~s}$ is computed. Indeed we consider rhythmical events of duration less then $5 \mathrm{~s}$ not classified as seizure (see [11] and references therein). Twenty Monte Carlo runs were performed for each of the parameters SBR, $\alpha$, $f_{o}(\alpha=0$ in that case), and SNR, within a prescribed range.

The detection signal, composed of ones and zeros, shows the occurence of rhythmical activity. The time locations of the ones depend on the detection scheme in use. A one is said to be an alarm. A good detection (GD) occurs when one alarm falls within one seizure interval. Multiple occurences of alarms during one seizure interval are considered as only one. An FD occurs when the alarm is not within any seizure interval. The total number of GDs and FDs are then counted. We define the GD rate (GDR) and FDR as

$$
\begin{aligned}
& \mathrm{GDR}=100 \times \frac{\mathrm{GD}}{\mathrm{SZ}_{>10}} \\
& \mathrm{FDR}=100 \times \frac{\mathrm{FD}}{\mathrm{GD}+\mathrm{FD}} .
\end{aligned}
$$

Actually, GDR is the percentage of true positive detection, and FDR is the percentage of false positive detections. Due to the use of a sliding window in each (LIU, GOTMAN, and SSA-MDL) method, ambiguity about the existence of rhythmical acivity occurs at the border of seizure patterns. For this reason, we have allowed a window margin of $5 \mathrm{~s}$ for the LIU and SSA-MDL methods and $10 \mathrm{~s}$ for the GOTMAN method to account for method-dependent border effects. The window margin is greater for GOTMAN because this method uses a wider sliding window (see Section IV-B).
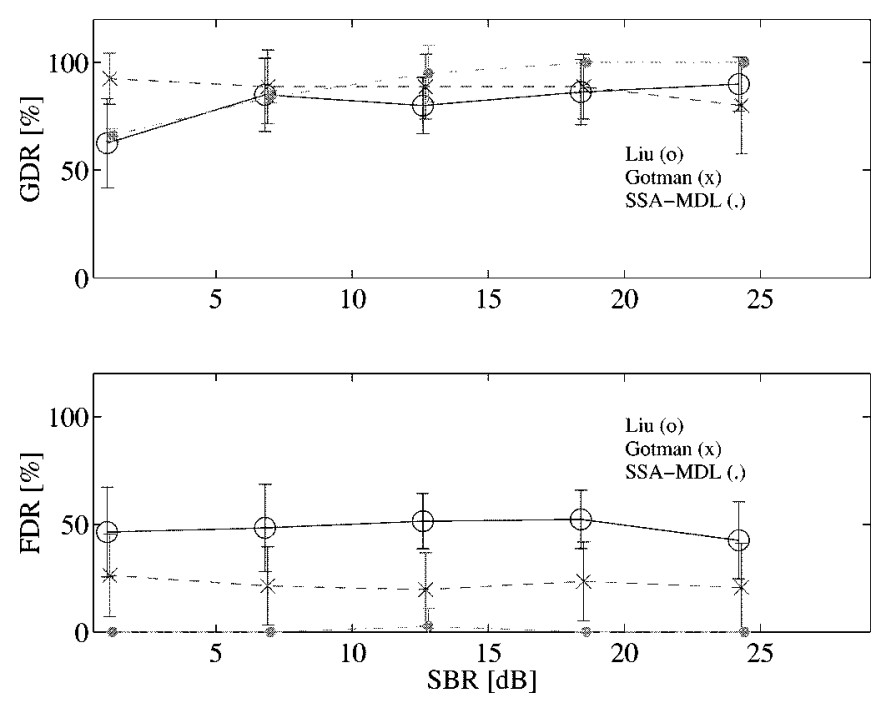

Fig. 5. Monte Carlo simulations for $1 \leq \mathrm{SBR} \leq 24 \mathrm{~dB}$.
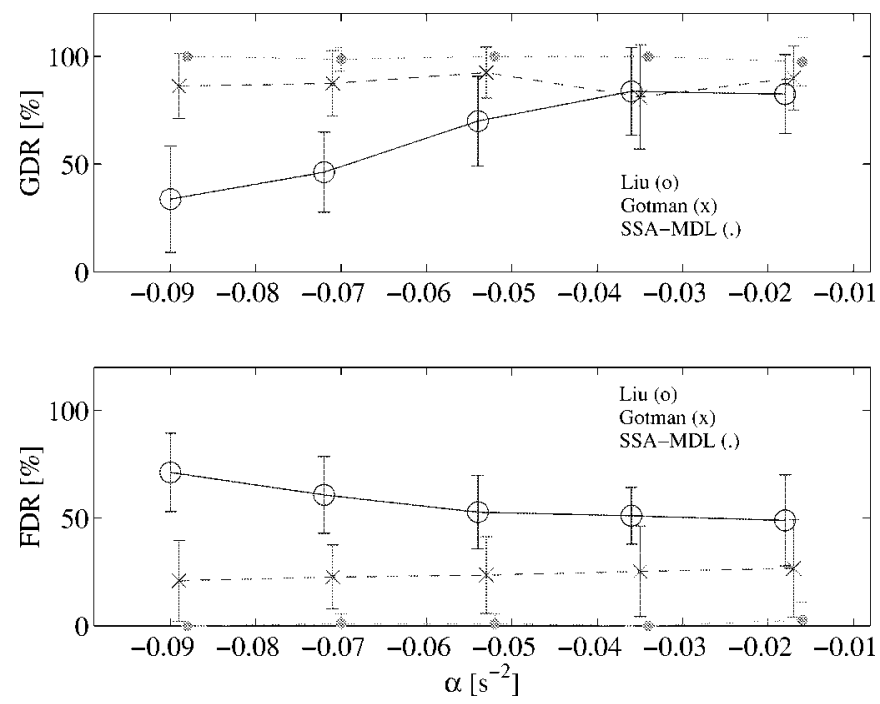

Fig. 6. Monte Carlo simulations for $-0.09 \leq \alpha \leq-0.018 \mathrm{~s}^{-2}$.

For the SSA-MDL method, we still have to select $n_{s}$. Supposing that the bandwidth $\Delta B$ of seizure signals varies from 1 $\mathrm{Hz}$ to $5 \mathrm{~Hz}$, we have $8<n_{s}<40$. In our experiments, we used $n_{s}=20$ for all the parameters $\left\{\alpha, f_{o}, \mathrm{SBR}, \mathrm{SNR}\right\}$.

Figs. 5, 6, and Fig. 8 display the results with mean and standard deviations. Next, we will discuss each figure independently.

\section{A. Mean Computation Time}

The mean computation times ${ }^{3}$ required to run detection methods are $1.15 \mathrm{~s}$ for LIU, $9.06 \mathrm{~s}$ for GOTMAN, and $7.9 \mathrm{~s}$ for SSA-MDL. The LIU algorithm clearely outperforms the other methods. This is due to the fact that the Liu's method does not allow any overlap between sliding windows and to the reduced complexity of the detection algorithm. GOTMAN and SSA-MDL have comparable performances.

\footnotetext{
${ }^{3}$ We have used a Pentium III 700-MHz machine with 256-MB RAM, and implementted the detection methods in Matlab.
} 


\section{B. Signal-to-Background Ratio (SBR)}

For this simulation, we fixed $f_{o}=1.42 \mathrm{~Hz}, \alpha=-0.015 \mathrm{~s}^{-2}$, $\mathrm{SNR}=11 \mathrm{~dB}$, and varied SBR $(1 \leq \mathrm{SBR} \leq 24 \mathrm{~dB})$. Results are shown in Fig. 5. We can observe that LIU has a lower mean GDR than GOTMAN and SSA-MDL for all SBR. Both LIU and SSA-MDL shows a drop in GDR for SBR $<7 \mathrm{~dB}$ while GOTMAN shows a re-markedly constant GDR. The SSA-MDL has a minimum GDR $=95 \%$, but greater than GOTMAN for $\mathrm{SBR} \geq 7 \mathrm{~dB}$ which is a far more smaller lower bound from our estimation of SBR on real data in Section III. LIU has the larger FDR, while GOTMAN has a greater FDR than SSA-MDL. With a maximum FDR $=2.6 \%$, SSA-MDL outperforms both LIU and GOTMAN. Standard deviations of GDR and FDR are almost the same for LIU and GOTMAN, while much greater than for SSA-MDL.

\section{Linear FM Slope $(\alpha)$}

Fig. 6 was obtained by fixing $f_{o}=1.42 \mathrm{~Hz}, \mathrm{SBR}=20 \mathrm{~dB}$, and $\mathrm{SNR}=11 \mathrm{~dB}$ and varying $\left.(-0.09 \leq \alpha \leq-0.018 \mathrm{~s}\}^{-2}\right)$. GOTMAN method provides an almost constant GDR, while LIU shows a strong linear decrease in GDR when $\alpha$ becomes more negative. The GDR behavior for LIU can be explained by the fact that the autocorrelation function of a linear FM signal does not exhibit the expected regular $T_{1}, \ldots, T_{4}$ intervals. As $\alpha$ decreases, the third and fourth peaks in the autocorrelation function tend to disappear. GOTMAN shows a greater or equal and almost constant GDR than LIU and much smaller FDR than LIU. The FDR for LIU increases significantly when $\alpha$ becomes more negative. SSA-MDL shows a better performance than both LIU and GOTMAN with a minimum GDR $=97 \%$, while showing an increasing FDR for highly nonstationary seizure with a maximum $\mathrm{FDR}=2.5 \%$.

\section{Frequency of the Seizure $\left(f_{0}\right)$, for the Stationary Case}

In this simulation, we fixed $\alpha=0 \mathrm{~s}^{-2}, \mathrm{SBR}=20 \mathrm{~dB}, \mathrm{SNR}=$ $11 \mathrm{~dB}$ and varied $f_{o}\left(0.5 \leq f_{o} \leq 1.7 \mathrm{~Hz}\right)$ to obtain results shown in Fig. 7. Both LIU and GOTMAN methods show a decrease in GDR when $f_{o}$ is decreased. This effect can be explained by the increased bias and variance of the autocorrelation function (and thus of the power spectrum density) estimates for low frequencies (the processing window is constant for all frequencies). LIU shows a lower or equal GDR than GOTMAN in any cases. For LIU, the FDR shows a significant decrease when $f_{o}$ is decreased, while GOTMAN shows an almost constant one. The FDR of LIU is larger than GOTMAN in any cases. SSA-MDL outperforms both LIU and GOTMAN with a constant GDR with a minimum of $\mathrm{GDR}=94 \%$ and a maximum $\mathrm{FDR}=2.6 \%$.

\section{E. Signal-to-Noise Ratio}

In this experiment, we fixed $\alpha=-0.015 \mathrm{~s}^{-2}, \mathrm{SBR}=20 \mathrm{~dB}$, and $f_{o}=1.42 \mathrm{~Hz}$ and varied SNR $(5 \leq \mathrm{SNR} \leq 17 \mathrm{~dB})$. The noise $n(t)$ is Gaussian and white. All the methods show an almost constant GDR and FDR. The GDR of LIU and GOTMAN are very similar (see Fig. 8). The FDR of LIU is much greater than the one of GOTMAN. SSA-MDL outperforms both LIU and GOTMAN with a constant GDR $=100 \%$ and a maximum $\mathrm{FDR}=1.6 \%$.
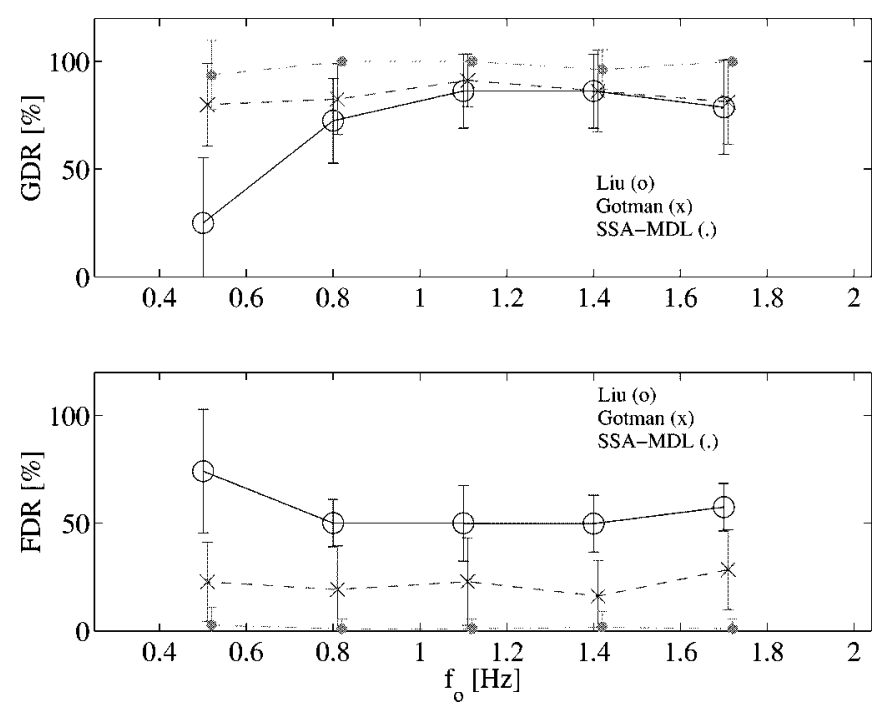

Fig. 7. Monte Carlo simulations for $0.5 \leq f_{o} \leq 1.7 \mathrm{~Hz}$.
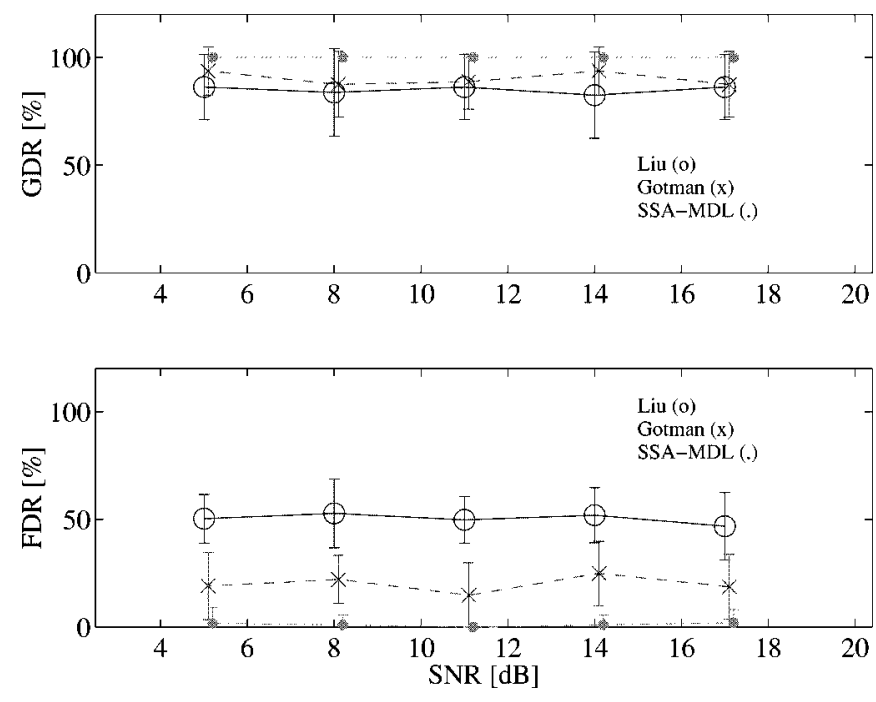

Fig. 8. Monte Carlo simulations for $5 \leq \mathrm{SNR} \leq 17 \mathrm{~dB}$.

\section{Performance Comparison on ExPerimental Data}

Seizure detection should be performed on all the recorded channel because the spatial location of the seizure is a priori unknown. Nevertheless, we have selected by hand one channel where electrical seizures occurs, and run the different detection schemes on this channel. Multichannel detection should be used in practice by serial or parallel processing.

Table I shows the result of the three detection schemes on real newborns and infants EEG seizure signals. All the EEG signals where normalized to have zero-mean and unit variance for further processing. For a sake of comparison with the Monte Carlo, all the EEGs where also normalized to have a duration of $T=8 \mathrm{~min}$. Additional background activity from the same recording was added at the begining of the EEG channels to meet this requirement and not introduces artificial artifacts.

The LIU method shows an average GDR $=59 \%$ and an average $\mathrm{FDR}=47 \%$. The GOTMAN method shows an average GDR $=73 \%$ and an average FDR $=29 \%$. 
TABLE I

Performance Results on Real EEG Data (a) LiU and GOTMAN. (b) SSA-MDL AND (SSA-MDL) $p$

\begin{tabular}{c||c|c|c|c}
\hline \multicolumn{1}{c||}{} & \multicolumn{2}{c|}{ LIU } & \multicolumn{2}{c}{ GOTMAN } \\
\cline { 2 - 5 } Baby Number & GDR & FDR & GDR & FDR \\
\hline 1 & 63 & 35 & 73 & 21 \\
\hline 2 & 54 & 45 & 68 & 23 \\
\hline 3 & 68 & 52 & 79 & 32 \\
\hline 4 & 51 & 58 & 73 & 41 \\
\hline
\end{tabular}

(a)

\begin{tabular}{c||c|c|c|c}
\hline \multicolumn{1}{c||}{} & \multicolumn{2}{c|}{ SSA-MDL } & \multicolumn{2}{c}{$(\text { SSA-MDL })_{p}$} \\
\cline { 2 - 5 } Baby Number & GDR & FDR & GDR & FDR \\
\hline 1 & 85 & 4 & 80 & 12 \\
\hline 2 & 96 & 6 & 85 & 14 \\
\hline 3 & 97 & 3 & 90 & 7 \\
\hline 4 & 95 & 5 & 92 & 15 \\
\hline
\end{tabular}

(b)

The SSA-MDL column refer to the use of SSA-MDL with preprocessing, while $\mathrm{SSA}_{-\mathrm{MDL}_{p}}$ is without the use of the preprocessing. Results show that the FDR decrease drastically with the preprocessing especially for the baby number 4 for which large bursts of short EEG rhythmical activities were observed, thus increasing the non-Gaussianity of the signals, and affecting the FDR of all the methods. The SSA-MDL method shows an average $\mathrm{GDR}=93 \%$ and an average $\mathrm{FDR}=4 \%$.

While the GDR of all methods are smaller than using the Monte Carlo, they are still in the same relationship. The FDR of all the methods showed higher values on real data than using the Monte Carlo, especially on highly non-Gaussian signals. This result demonstate the necessity of a preprocessing techniques prior to applying any detection methods.

\section{DISCUSSION}

We proposed a new EEG seizure detection scheme based on SSA and a model order selection criterion originally developed by Rissanen. The use of the Rissanen's model selection criterion enabled us to design a data- and analysts-independent detector. Preprocessing the EEG using a recently proposed seizure model enable to use the MDL criterion in an optimal way, and to reduce the number of false alarms. Monte Carlo simulations on the three detection schemes have been performed. The results show the following.

1) The LIU method is computationally efficient.

2) The LIU method gives lower GDR and higher FDR than both SSA-MDL and GOTMAN.

3) The GOTMAN method is robust against SBR, while LIU and SSA-MDL are not for SBR $<6 \mathrm{~dB}$. It is to be noted that $\mathrm{SBR}<6 \mathrm{~dB}$ is quite unusual and have not been reported in our work.
4) LIU is very sensitive to the frequency $f_{o}$, while SSA-MDL and GOTMAN are more robust.

5) LIU is very sensitive to the nonstationarity parameter $\alpha$, while GOTMAN and SSA-MDL are more robust.

6) All methods are robust against Gaussian white noise.

The most critical factor affecting the performances of the different schemes is the nonstationarity represented by $\alpha$. This is not surprizing because all three methods are dedicated to stationary time series analysis even if they are processed by sliding windows. The proposed SSA-MDL detector has better performances in terms of GDR and FDR than the two other methods. The poorest performance of the SSA-MDL scheme has been achieved for the lowest SBR. One possible drawback of this method is the need to perform SVD decomposition on a trajectory matrix, hence limiting its application to off-line processing. To overcome this limitation, we have also proposed a real-time implementation of this method using adaptive algorithms [27], [28].

Results on real data show that SSA-MDL is a performant method compared with GOTMAN and LIU. The average GDR and FDR are lower than in the Monte Carlo, but still very good. The GDR of GOTMAN and SSA-MDL are quite comparable, but the very low FDR of SSA-MDL is a landmark of potential clinical assessment.

\section{CONCLUSION}

A new infant EEG seizure detection scheme based on SSA and model selection was presented. Using synthetic data, we were able to compare the performance of our method with that of two previously published techniques. Performance comparison was also conducted on a set of four infants showing signs of clinical and electrical seizures. The SSA-MDL method was shown to outperform the other two, especially in terms of FDR. Adaptive SSA-MDL is currently under investigation and should further improve the performance of the new detection scheme concerning both highly nonstationary environment and its real-time aspects.

Again, we would like to emphasize that the computer-based detection is a support for the clinician and does not provide the ultimate answer to the seizure detection problem, particularly for newborn and infant EEGs.

\section{ACKNOWLEDGMENT}

The authors would like to thank J. Richmond and L. Welch of the Neuroscience Department, Royal Children's Hospital, who have performed the recordings, and Dr. C. Burke who has segmented the seizure EEG signals. They would also like to acknowledge the assistance of M. Keir, F. Kearney, and Ms. J. Deviney for obtaining the seizure EEG recordings at the ICU of the Royal Women's Hospital.

\section{REFERENCES}

[1] J. J. Volpe, Neurology of the Newborn. Philadelphia, PA: Saunders, 1997.

[2] M. C. Toet, I. Hellstrom-Westas, F. Groenendaal, P. Eken, and I. S. de Vries, "Amplitude integrated EEG 3 and 6 hours after birth in full term neonates with hypoxic-ischaemic encephalopathy," Arch. Dis. Child Fetal Neonatal Educ., vol. 81, pp. F19-F23, 1999. 
[3] J. C. Rowe, G. L. Holmes, J. Hafford, D. Baboval, S. Robinson, A. Philipps, T. Rosenkrantz, and J. Raye, "Prognostic value of the electroencephalogram in term and preterm infants following neonatal seizures," Electroencephalogr. Clin. Neurophysiol., vol. 60, pp. $183-196,1985$

[4] A. Liu, J. S. Hahn, G. P. Heldt, and R. W. Coen, "Detection of neonatale seizures through computerized EEG analysis," Electroencephalogr. Clin. Neurophysiol., vol. 82, pp. 363-369, 1992.

[5] J. Gotman, D. Flanagan, B. Rosenblatt, A. Bye, and E. Mizrahi, "Evaluation of an automatic seizure detection method for the newborn EEG," Electroencephalogr. Clin. Neurophysiol., vol. 103, pp. 363-369, 1997.

[6] J. Rissanen, Stochastic Complexity in Statistical Inquiry, Singapore: World Scientific, 1989

[7] M. Wax and T. Kailath, "Detection of signals by information theoretic criteria," IEEE Trans. Acoust, Speech, Signal Processing, vol. ASSP-33, pp. 387-392, 1985

[8] C. T. Lombroso, "Neonatal EEG polygraphy in normal and abnormal newborns," in Electroencephalography, Basic Principles, Clinical Applications and Related Fields, E. Niedermeyer and F. H. Lopes da Silva, Eds. Baltimore, MD: Williams \& Wilkins, 1993, ch. 48, pp. 802-875.

[9] H. P. Zaveri, W. J. Williams, D. Iasemidis, and J. C. Sackellares, "Time-frequency representation of electrocorticograms in temporal lobe epilepsy," IEEE Trans Biomed. Eng., vol. 39, pp. 502-509, May 1992.

[10] P. J. Franaszczuk, G. K. Bergey, P. J. Durka, and H. M. Eisenberg, “Timefrequency analysis using matching pursuit algorithm applied to seizure originating from the mesial temporal lobe," Electroencephalogr. Clin. Neurophysiol., vol. 106, pp. 513-521, 1998.

[11] P. Celka, B. Boashash, and P. Colditz, "preprocessing and time-frequency analysis of newborn EEG seizures," IEEE Eng. Med. Biol Mag., vol. 20 , pp. 30-39, 2001.

[12] L. Ingber, "Statistical mechanics of neocortical interactions: Multiple scales of EEG," in Frontier Science in EEG, Continuous Waveform Analysis (EEG Suppl. 45), R. M. Dasheiff and E. D. J. Vincent, Eds. Berlin, Germany: Elsevier, 1996.

[13] P. Celka and P. Colditz, "Nonlinear nonstationary Wiener model of infant EEG seizures," IEEE Trans. Biomed. Eng., to be published.

[14] R. J. Ellingson, "Optimal recording techniques: Neonates and infants," in Clinical Neurophysiology of Epilepsy: EEG Handbook, J. A. Wada and R. J. Ellingson, Eds. Berlin, Germany: Elsevier Science, 1990, vol. 4, ch. 3, pp. 79-88.

[15] M. Roessgen, A. M. Zoubir, and B. Boashash, "Seizure detection of newborn EEG using a model-based approach," IEEE Trans. Biomed. Eng., vol. 45, pp. 673-685, June 1998.

[16] F. H. Lopes da Silva, A. Hoeks, H. Smits, and L. H. Zetterberg, "Model of brain rhythmic activity," Kybernetik, vol. 15, pp. 27-37, 1974

[17] "Postgraduate Lecture in Mathematical Ingeneering," Swiss Fed. Inst. Technol., Lusanne, Switzerland, 1998.

[18] J. Gotman, J. Zhang, J. Rosenblatt, and R. Gottesman, "Automatic seizure detection in newborns and infants," in Proc. IEEE EMBS'95, vol. 2, pp. 913-914.

[19] R. Vautard, P. Yiou, and M. Ghil, "Singular-spectrum analysis: A toolkit for short, noisy chaotic signals," Physica D, vol. 58, pp. 95-126, 1992.

[20] D. S. Broomhead and G. P. King, "Extracting qualitative dynamics from experimental data," Physica D, vol. 20, pp. 217-236, 1986.

[21] A. I. Mees, P. E. Rapp, and L. S. Jennings, "Singular-value decomposition and embedding," Phys. Rev. A, vol. 36, pp. 340-346, 1987.

[22] A. Passamante, T. Hediger, and M. Gollub, "Fractal dimension and local intrinsic dimension," Phys. Rev. A, vol. 39, p. 3640, 1989.

[23] M. Palŭs and I. Dvorak, "Singular-value decomposition in attractor reconstruction: Pitfalls and precautions," Physica D, vol. 55, pp. 221-234, 1992.

[24] A. Mineva and D. Popivanov, "Method for single-trial readiness potential identification, based on singular spectrum analysis," J. Neurosci. Meth., vol. 68, pp. 91-99, 1996.

[25] R. Vetter, J.-M. Vesin, N. Virag, P. Celka, and U. Scherrer, "Observer of autonomic cardiac outflow based on blind source separation of ECG parameters," IEEE Trans. Biomed. Eng., vol. 47, pp. 578-582, May 2000.

[26] R. Vetter, "Extraction of efficient and characteristic features of multidimensional time series: Application to the human cardiovascular system," Ph.D. dissertation, Swiss Fed. Inst. Technol., Lusanne, Switzerland, 1999.
[27] P. Celka and P. Colditz, "Time-varying statistical dimension analysis with application to newborn scalp EEG seizure signals," Med. Eng. Physics, vol. 24, no. 1, pp. 1-8, 2002.

[28] — "Time-varying statistical complexity measures with application to EEG analysis and segmentation," presented at the EMBS2001, Istanbul, Turkey, 2001

[29] R. Hornero, P. Espino, A. Alonso, and M. Lopez, "Estimating complexity from EEG background activity of epileptic patients," IEEE EMB Mag., vol. 18, pp. 73-79, 1998.

[30] K. Lehnertz and C. E. Elger, "Spatio-temporal dynamics of the primary epileptogenic area in temporal lobe epilepsy characterized bu neuronal complexity loss," Electro. Clin. Neurol., vol. 95, pp. 108-117, 1995.

[31] J. Theiler and P. E. Rapp, "Re-examination of the evidence for lowdimensional nonlinear structure in the human electroencephalogram," Electro. Clin. Neurol., vol. 98, pp. 213-222, 1996.

[32] G. K. Bergey and P. J. Franaszczuk, "Epileptic seizures are characterized by changing signal complexity," Electroencephalogr. Clin. Neurophysiol., vol. 112, pp. 241-249, 2001.

[33] J. Gotman, D. Flanagan, J. Zhang, and B. Rosenblatt, "Automatic seizure detection in the newborn: Methods and initial evaluation," Electroencephalogr. Clin. Neurophysiol., vol. 103, pp. 356-362, 1997.

[34] J. Gotman, J. R. Ives, and P. Gloor, "Automatic recognition of interictal epileptic activity in prolonged EEG recordings," Electroencephalogr. Clin. Neurophysiol., vol. 46, pp. 510-520, 1979.

[35] J. Gotman, "Automatic seizure detection: Improvements and evaluation," Electroencephalogr. Clin. Neurophysiol., vol. 76, pp. 317-324, 1990

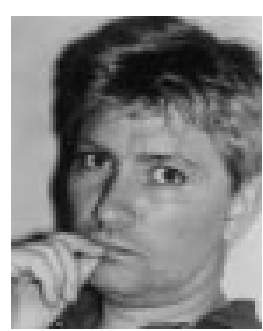

Patrick Celka (M'96) received the M.Sc. degree in physical sciences from the Catholic University of Louvain-la-Neuve, Louvain-la-Neuve, Belgium, in 1987. He received the M.S. in information and signal processing and the Ph.D. (Circuits and Systems Chair with Prof. M. Hasler) degrees from the Swiss Federal Institute of Technology (EPFL), Lusanne, Switzerland, in 1993 and 1995, respectively.

He worked as a Senior Research Assistant at the Signal Processing Laboratory of Prof. M. Kunt at EPFL from 1995 to 1999 . He was involved in neuro-cardiovascular system and signal modeling and analysis. From 1999 to 2001, he was leading a biomedical project dealing with the automatic detection/classification of EEG seizures in the newborn with the Signal Processing Research Centre, Queensland University of Technology, Brisbane, Australia. He is currently with the Systems Engineering Division of the Swiss Center for Electronics and Microtechnology, Neuchâtel, Switzerland. His fields of research include nonlinear dynamical systems theory, nonlinear signal and system modeling and identification, chaos theory and its application, nonlinear adaptive algorithms, and biomedical engineering.

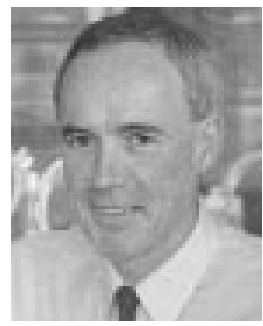

Paul Colditz received the M.S. and Ph.D. degrees from Oxford University, Oxford, U.K.

$\mathrm{He}$ is Professor of Perinatal Medicine, University of Queensland and director of the Perinatal Research Centre at the Royal Women's Hospital, Brisbane, Australia. $\mathrm{He}$ is a Specialist Paediatrician and Neonatologist. His interest in caring for babies and their families has led to undertaking and fostering multidisciplinary research of the events before, during, and shortly after birth which affect the health of individuals for the remainder of their lives. He directs a research program aimed at advancing the health of mothers and babies with the central focus being the development of the brain and, in particular, the prevention of death and brain damage in unborn and newborn babies. His research projects encompass fetal monitoring, cerebral palsy, birth asphyxia, mental retardation, intraventricular haemorrhage, periventricular leucomalacia, and sudden infant death syndrome. A related research interest is the development, evaluation, and clinical testing of new biomedical technologies. 